\title{
Effects and cost-effectiveness of a guideline-oriented primary healthcare hypertension management program in Beijing, China: results from a 1-year controlled trial
}

\author{
Xin Wang ${ }^{1,10}$, Weiqin $\mathrm{Li}^{2,10}$, Xian $\mathrm{Li}^{3}$, Ning $\mathrm{An}^{1,11}$, Hao Chen ${ }^{4}$, Stephen Jan ${ }^{5}$, Guanghua Ming ${ }^{1}$, Qi Hua ${ }^{1}$, \\ Xiaowei Yan $^{6}$, Ningling $\mathrm{Sun}^{7}$, Dong $\mathrm{Zhao}^{8}$ and Yangfeng $\mathrm{Wu}^{3,9}$
}

Hypertension control rates are unacceptably low in China. The present study demonstrates if a customized, guideline-oriented training program can cost-effectively improve hypertension management in primary healthcare. Four typical community health centers in Beijing were selected and randomized to intervention or control (one urban and one rural each). A sample of 140 patients with hypertension and blood pressure uncontrolled was recruited from each center. Primary healthcare providers in intervention centers provided management to the recruited patients for 1 year after receiving training with customized hypertension management guidelines, and primary healthcare providers in control provided with usual care. Intention-to-treat analysis showed that hypertension control (systolic blood pressure (SBP) $<140 \mathrm{~mm} \mathrm{Hg}$ and diastolic blood pressure (DBP) $<90 \mathrm{~mm} \mathrm{Hg})$ ) rate was significantly higher in interventions than controls at month $3(42.1 \%$ vs. $34.3 \%$ in urban and $30.7 \%$ vs. $10.0 \%$ in rural centers) and the trend increased to month $12(70.7 \%$ vs. $40.0 \%$ in urban and $72.9 \%$ vs. $27.9 \%$ in rural); $P$-values by logistic mixed model were all $<0.001$ for both urban and rural after adjustment for baseline multiple variables including blood pressure. Mean reductions of SBP and DBP were significantly larger in interventions. The intervention was cost-saving, with an average incremental cost-saving of US\$20.3 per patient in urban sites and $\$ 7.0$ per patient in rural sites. Corresponding results from per-protocol analysis were very similar. The customized, guideline-oriented hypertension management program in primary healthcare in China effectively improved blood pressure control and was cost-saving.

Hypertension Research (2013) 36, 313-321; doi:10.1038/hr.2012.173; published online 15 November 2012

Keywords: cost-effectiveness; evaluation; guideline; intervention

\section{INTRODUCTION}

Cardiovascular disease is the leading cause of death worldwide, including in countries such as China. ${ }^{1-3}$ Hypertension accounts for the highest attributable risk for loss of healthy life years from cardiovascular death. ${ }^{4,5}$ According to the 2002 National Nutrition and Health Survey, the prevalence of hypertension was $18 \%$ in adults aged over 18 years, an increase of 31\% compared with that in 1991 when it was estimated that about 160 million people in China were hypertensive. ${ }^{6-8}$

In contrast, levels of awareness, treatment and control of hypertension were quite low, at $24 \%, 19 \%$ and $5 \%$, respectively, in $2002 .^{7}$ The ratio of control to treatment was 1:4. In comparison, the awareness, treatment and control of hypertension in the
United States were $70 \%, 59 \%$ and $34 \%$, respectively, and the ratio was higher than $1: 2 .{ }^{9}$ Although the mortality of cardiovascular disease had dropped by more than $50 \%$ since the early 1970s in the United States, mortality has kept increasing in China and is expected to increase in the next 20 years if interventions remain unchanged. 7,10

To involve primary healthcare providers in China in the control of hypertension and to overcome their low technical capacity, a simplified, pragmatic, customized and evidence-based guideline, 'The Primary Healthcare Practice Guidelines for Hypertension Prevention and Control', was developed and adopted by the Chinese professional associations, the Chinese Center for Disease Control and Prevention and the Ministry of Health. ${ }^{11}$ This paper reports on the

${ }^{1}$ Fuwai Hospital, Chinese Academy of Medical Sciences, Beijing, China; ${ }^{2}$ Tianjin Women's and Children's Health Center, Tianjin, China; ${ }^{3}$ The George Institute for Global Health, Beijing, China; ${ }^{4}$ Beijing Hospital, Beijing, China; ${ }^{5}$ The George Institute for Global Health, Sydney, NSW, Australia; ${ }^{6}$ Peking Union Medical College Hospital, Beijing, China; ${ }^{7}$ Peking University People's Hospital, Beijing, China; ${ }^{8}$ Beijing An Zhen Hospital of the Capital University of Medical Sciences, Beijing, China and ${ }^{9}$ Department of Epidemiology and Biostatistics, Peking University School of Public Health, Beijing, China

${ }^{10}$ These authors contributed equally to this work.

${ }^{11}$ Current address: Los Angeles County Department of Public Health, Tobacco Control and Prevention Program, Los Angeles, CA, USA.

Correspondence: Dr Y Wu, Department of Epidemiology and Biostatistics, Peking University School of Public Health, No. 38 Xueyuan Road, Haidian District, Beijing 100191, China. 
first study conducted in China, to evaluate the effectiveness of applying the guidelines in practice.

\section{METHODS}

\section{Study sites}

Four community health centers in Beijing, two from urban districts and two from rural districts, were selected as representative in terms of social and economic development, through recommendations from the local health authorities. The study sites were stratified by urban and rural because of the very large social and economic differences that exist.

\section{Patients}

We recruited 140 uncontrolled hypertensive patients in each community health center for the study. All primary healthcare providers were trained to use a simple tabulated case management record (CMR) to document the initial clinical visit and each subsequent follow-up visit. All patients received followup every month until the end of month 12 .

Patients had to meet the following criteria: they should be 18 years of age or older, should have primary hypertension with uncontrolled blood pressure (systolic blood pressure (SBP) $\geqslant 140 \mathrm{~mm} \mathrm{Hg}$ or diastolic blood pressure (DBP) $\geqslant 90 \mathrm{~mm} \mathrm{Hg}$, regardless of anti-hypertension medication use), should be managed mainly in the community health centers, should be willing to participate and sign the consent form, should not plan to move away from the community in the following year and should not have any severe diseases or mental illnesses that could significantly affect his/her commuting to the center for regular follow-up visits or have normal communications with primary healthcare providers. The study protocol was approved by the Ethics Committee of Cardiovascular Institute and Fuwai Hospital, Chinese Academy of Medical Sciences. The written informed consent was obtained from all study patients.

\section{Intervention}

The intervention was randomly assigned to one of the urban centers and one of the rural centers. Primary healthcare providers in the intervention centers received an initial four and a half day group-training lectures in the Primary Healthcare Practice Guidelines for Hypertension Prevention and Control (referred to as 'Practice Guidelines' below) and four subsequent individualbased consolidated training, one per quarter, based on the actual cases. The contents of the Practice Guidelines included detection, evaluation, nonpharmaceutical and pharmaceutical treatment, follow-up and management, two-way referral, prevention and health education for hypertension. ${ }^{12}$ Briefly, primary healthcare providers were asked to treat the patient's blood pressure to the target $(<140 / 90 \mathrm{~mm} \mathrm{Hg})$. Low-cost generic medications were recommended and low-dose combination use of anti-hypertension medications were encouraged. Lifestyle consultations were also included, emphasizing on low-salt diet, regular exercise, weight reduction and smoking cessation at all visits. All patients were asked to come to the centers once per month for regular follow-up visits, with blood pressure being measured at all visits and medical treatments adjusted accordingly (titrated to a higher dose or added onto another medication). Lifestyle advice was reinforced if a patient's blood pressure targets were not reached. Patients were to be referred to the specialists if there were any cardiovascular disease signs, symptoms and situations presented during the follow-up that the primary healthcare provider felt unable to deal with. Primary healthcare providers in the control groups received no training and provided usual care to their patients.

\section{Sample size}

The sample size estimation was based on the blood pressure control rate, with an estimated rate of $20 \%$ in the control groups and $20 \%$ absolute increase in the intervention groups at the end of the study. ${ }^{6,7,12}$ A minimum sample size of 108 participants in each community was required with a power of $90 \%$. Taking into account the loss to follow-up, we required each center to recruit 140 patients.

\section{Measurements and outcomes}

Information was obtained using the tabulated CMR filled out by primary healthcare providers at a monthly basis, including demographic information, blood pressure measurements, risk factors, new cardiovascular and renal diseases, medical treatments and lifestyle advices. Blood pressure was measured using standardized methods in every follow-up visit by the primary healthcare providers using a validated sphygmomanometer (model: Yuyue GB305393, Shanghai Yu Yue Medical Equipment Co., Ltd., Shanghai, China). ${ }^{11,13}$

The primary outcome was blood pressure control rate, specifically, the percentage of patients with SBP $<140 \mathrm{~mm} \mathrm{Hg}$ and DBP $<90 \mathrm{~mm} \mathrm{Hg}$ among all patients. The secondary outcomes included the reduction of SBP and DBP from baseline. Costs and cost-effectiveness were also assessed. Information on anti-hypertension medication use, including the brand names and doses, was collected from the doctor's prescriptions. Information on non-pharmaceutical treatment was also collected from the tabulated CMRs of the lifestyle advice given by the primary healthcare providers, and the use of both pharmaceutical and non-pharmaceutical treatments was analyzed as process indicators to help understand the possible mechanisms that influence the control of blood pressure in the intervention groups. Cardiovascular and renal diseases refer to any incident that may cause damage to the heart or vessels, including stroke, coronary heart disease, diabetes and renal disease.

We conducted an economic evaluation using a health sector perspective. The overall cost in each group included the costs for drugs, time spent by the primary healthcare providers (taking care of the participating patients and attending the training sessions), training (compensation to the trainers, training materials, the facilities for training and transportation for trainers to travel to the community health centers), administration (salary compensation to the health administrator, called 'Project Administrator' in the study and the transportation to the sites for project monitoring) and the tabulated CMRs. Given the health sector perspective, we did not include the patient's indirect costs, such as time for seeing the doctor and transportation from home to the community health centers/clinics. Drug cost was calculated by the local market price of the drug multiplied by the dose per day and days of treatment, and all costs were then converted to the US dollars using the exchange rate in year 2002 (one US dollar equals to 8.2772 Chinese Yuan).

\section{Statistical analysis}

To simplify the data analysis and avoid too many missing values induced by missed follow-ups, we decided to analyze the blood pressure data on a quarterly basis rather than a monthly basis, and use their measurements that were most close to the time point of month 3,6,9 and 12 .

Both the intention-to-treat (ITT) and per-protocol (PP) analyses were conducted to assess the efficacy of the intervention. ITT analysis used data from all patients $(140 \times 4=560)$, with missing values of blood pressure readings replaced by the value taken in the previous visit.

During the study period ( 12 months), 3 patients died and 9 moved out of the residential area, but loss to follow-up were balanced between intervention and control groups. Another 112 patients did not show up to at least one of the four quarterly follow-up visits, and had one or more missing values of blood pressure measurements. PP analysis only included the 436 patients (77.9\%) who had blood pressure measured at all the four quarterly visits (Figure 1).

Statistical analyses were conducted using SAS 9.2 software package (Peking University Health Science Center, Beijing, China), and $P<0.05$ were considered statistically significant. For the primary outcome of blood pressure control rates, the logistic mixed model was used to test the overall intervention effect and the linear trend over time, adjusting for possible confounding factors such as baseline blood pressures, age, body mass index and the duration of hypertension, which accounted for the cluster effects and potential correlation within patient from the repeat measurements at months 3, 6, 9 and 12. The comparison of the reduction on SBP and DBP levels between the intervention and control groups was implemented using the general linear mixed model, which accounted for the cluster effects and potential correlation from repeat measurements within each individual patient. The interaction terms of the intervention and time were introduced into both models so as to check if the trend over time differed by intervention groups. The $P$-values from multiple comparison of the control rate or blood pressure reduction were given after 

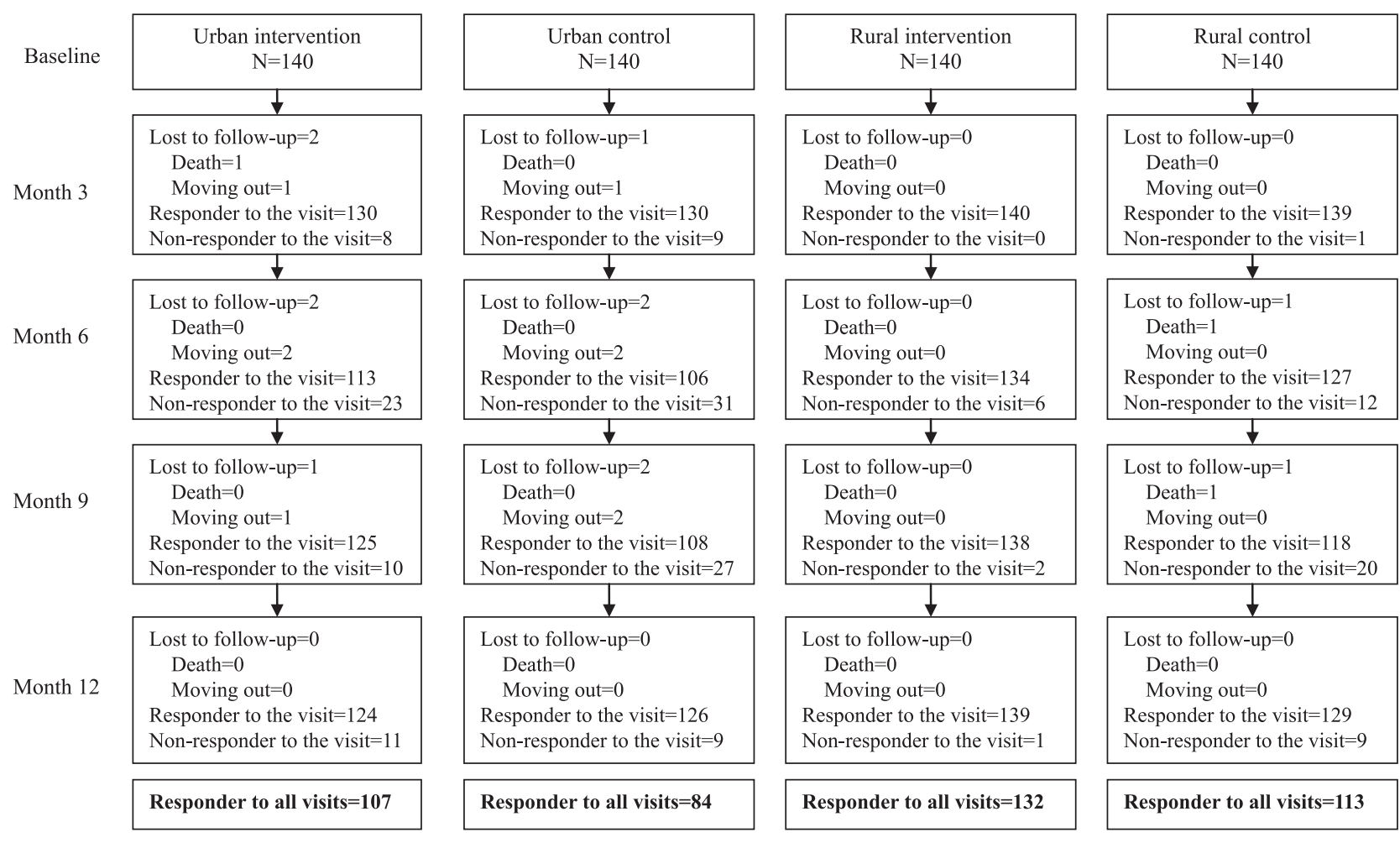

Figure 1 Flow chart of study patients during the follow-up.

correction by the Bonferroni method. We reported unadjusted and adjusted blood pressure control rates and mean reduction of blood pressure, together with the corresponding 95\% confidence intervals, between the intervention group and the control group by urban and rural, adjusted for age, body mass index, SBP/DBP and the duration of hypertension.

To measure the changes in non-pharmaceutical treatments over the intervention period, we used data at month 1 and month 12 from the CMR, because no such data could be available before the intervention according to the study design.

A Student's $t$-test was used to compare the means and the $\chi^{2}$-test to compare the rates between intervention and control groups at baseline, and for the comparisons in secondary analyses.

\section{RESULTS}

\section{Baseline characteristics}

As shown in Table 1, patients in the intervention and control groups were similar in gender, alcohol drinking and tobacco smoking in both urban and rural centers. Patients in the intervention groups had better education attainment and lower SBP and DBP, but more history of disease, particularly coronary heart disease. In addition, patients in the urban intervention were older and had less obesity than in the urban control group, whereas no such differences were observed in their rural counterparts.

\section{The effects on blood pressure control}

The results from logistic mixed model as in Table 2 showed that the overall effect of intervention were significant in both urban and rural centers after adjusted for SBP at baseline, age, body mass index and duration of hypertension (all $P$-values $<0.01$ ). The blood pressure control rate increased with time in both the intervention and control groups (most $P$-values $<0.0001$ ), except for the control group in urban sites, but the magnitude of increasing of control rate with time was greater in intervention group than in control group (all $P$-values $<0.01$ ). This resulted in increasing wider differences in blood pressure control rates between the intervention and control groups so that the differences turned significant from 9 to 12 month in the urban, and from 3 month in the rural group. The results from PP and ITT analyses are very similar.

\section{The effects on SBP and DBP reduction}

The reduction in SBP and DBP levels appeared to linearly increase over time in both the urban and rural group, no matter whether in the intervention or control groups (most $P$-values for trend test $<0.001$ ), except for the DBP reduction of control group in the urban group ( $P=0.7559$ for PP analysis). On the other hand, the magnitude of increase on blood pressure reduction over time appeared larger in the intervention than in control group, both in urban and rural, with the exception of SBP in the urban group. The overall effects of intervention on SBP or DBP were significant in both urban and rural at PP analysis. For urban, the difference became statistically significant at month 9 and 12 for SBP and, since month 3 and afterwards for DBP. For rural, the difference became statistically significant at month 6 and afterwards for SBP and at month 9 and 12 for DBP. Similar to the trend of blood pressure control rate, the blood pressure reduction became increasingly larger in intervention groups but not in controls, so that the difference between the intervention and control groups became much wider at the end of study (Table 3). Again, PP and ITT analyses showed very similar results.

\section{Economic analysis}

As shown in Table 4, the average annual cost of per patient in the intervention group were significantly less than those in the control group, in both urban and rural settings. The higher training, 
Table 1 Baseline characteristics of study patients in intervention and control groups

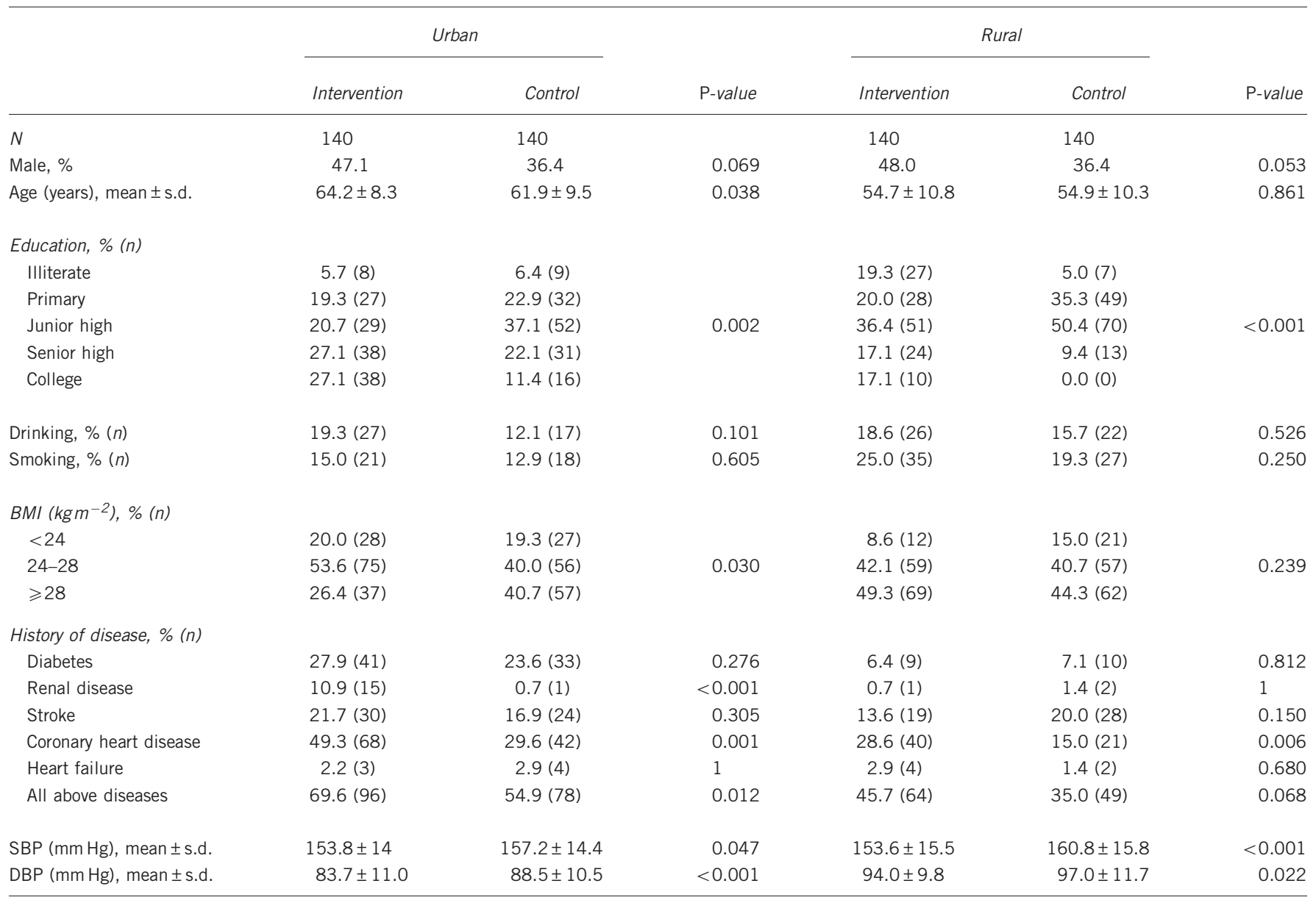

Abbreviations: BMI, body mass index; DBP, diastolic blood pressure; SBP, systolic blood pressure.

administration and salary costs of the primary healthcare providers were more than offset by the lower drug costs incurred in this group in both urban and rural settings (ITT analysis: by $\$ 20.3$ and $\$ 7.0$ per patient, respectively). Thus, the intervention is found to be costsaving and allied with the positive-effectiveness findings; the costeffectiveness of the intervention can be seen to be dominant over usual care.

\section{Secondary analysis}

We further analyzed the changes in use of non-pharmaceutical and pharmaceutical treatments provided by the primary healthcare providers to better understand the mechanisms that led to the improvement of blood pressure control in the intervention groups. As PP and ITT analyses showed very similar results, we only used PP analysis for this purpose.

At month 1, the percentage of patients on non-pharmaceutical treatment was already significantly higher in the intervention groups than in controls $(P<0.001)$, in both urban and rural centers (Table 5). From month 1 to month 12 , the percentage of patients on non-pharmacological treatments increased almost exclusively in the intervention groups, but remained unchanged in controls (Figure 2).
All patients had been taking pharmaceutical treatments since the beginning of the study. Generally, there was no significant change in the use of each type of anti-hypertension drugs, except for the calcium antagonist that increased significantly in the rural intervention, and $\beta$-blocker that decreased significantly in the urban control. The most striking change in the pharmaceutical treatment was a significant decrease in the number of patients on a single-drug therapy, but those on a two-drug combination increased significantly in both urban and rural interventions (Figure 2).

During the 1 year of follow-up, there were 15 new cardiovascular and renal diseases, 3 in the urban intervention group, 6 in the urban control group, 4 in the rural intervention group and 2 in the rural control group. As not many events would be expected in such a smallscale study, we did not try to perform the statistical test for the difference.

\section{DISCUSSION}

Before China established its community health services, hypertension was not considered the responsibility of primary healthcare providers, mainly because of their insufficient medical training. Similar to other developing countries, hypertension management in China had predominantly been hospital-based and specialist-focused. The majority of people with hypertension had limited access to care and this 
Table 2 Crude percentage and adjusted percentage $(95 \% \mathrm{Cl})$ of patients with blood pressure under control in intervention and control groups, urban and rural, at different time of follow-up, adjusting for the baseline blood pressure, age, BMI and duration of hypertension

\begin{tabular}{|c|c|c|c|c|c|c|c|c|}
\hline \multirow[b]{3}{*}{ Month of follow-up } & \multicolumn{4}{|c|}{ Urban } & \multicolumn{4}{|c|}{ Rural } \\
\hline & \multicolumn{2}{|c|}{ Intervention $(107 / 140)^{a}$} & \multicolumn{2}{|r|}{ Control (84/140) } & \multicolumn{2}{|c|}{ Intervention (132/140) } & \multicolumn{2}{|c|}{ Control (113/140) } \\
\hline & $\%$ & Adjusted \% (95\% Cl) & $\%$ & Adjusted \% (95\% Cl) & $\%$ & Adjusted \% (95\% Cl) & $\%$ & Adjusted \% (95\% Cl) \\
\hline \multicolumn{9}{|l|}{$P P$ analysis } \\
\hline 3 & 45.8 & $41.0(29.8,53.1)$ & 38.1 & $34.8(23.2,48.6)$ & 30.3 & $16.5(10.4,25.1)$ & 10.6 & $4.3(2.2,8.4)^{*}$ \\
\hline 6 & 57.0 & $56.9(44.6,68.3)$ & 47.6 & $49.0(35.3,62.8)$ & 50.0 & $45.3(33.2,58.0)$ & 22.1 & $14.7(8.6,23.9)^{* *}$ \\
\hline 9 & 72.9 & $78.1(67.8,85.8)$ & 52.4 & $56.2(42.2,69.3)$ & 68.2 & $75.9(65.1,84.2)$ & 29.2 & $24.4(15.4,36.4)^{* *}$ \\
\hline 12 & 74.8 & $80.3(70.5,87.4)$ & 47.6 & $49.0(35.3,62.8)^{* *}$ & 73.5 & $82.9(73.9,89.3)$ & 27.4 & $21.7(13.5,33.2)^{* *}$ \\
\hline$P_{\text {trend }}$ & & $<0.0001$ & & 0.0455 & & $<0.0001$ & & $<0.0001$ \\
\hline$P_{\text {effect }}$ & \multicolumn{4}{|c|}{0.0089} & \multicolumn{4}{|c|}{$<0.0001$} \\
\hline$P_{\text {interaction }}$ & \multicolumn{4}{|c|}{0.0017} & \multicolumn{4}{|c|}{0.0001} \\
\hline \multicolumn{9}{|l|}{ ITT analysis } \\
\hline 3 & 42.1 & $33.6(24.1,44.8)$ & 34.3 & $28.2(19.4,39.0)$ & 30.7 & $16.4(10.4,24.8)$ & 10.0 & $3.8(2.0,7.0)^{* *}$ \\
\hline 6 & 53.6 & $51.4(39.8,62.8)$ & 40.7 & $38.3(27.8,50.1)$ & 50.0 & $44.9(33.0,57.4)$ & 21.4 & $13.6(8.3,21.6)^{* *}$ \\
\hline 9 & 70.0 & $76.5(66.6,84.2)$ & 43.6 & $43.1(32.0,55.0)^{* *}$ & 67.1 & $74.6(63.6,83.1)$ & 27.1 & $21.2(13.7,31.4)^{* *}$ \\
\hline 12 & 70.7 & $77.5(67.8,84.9)$ & 40.0 & $37.2(26.8,48.9)^{* *}$ & 72.9 & $82.4(73.4,88.8)$ & 27.9 & $22.3(14.5,32.7)^{* *}$ \\
\hline$P_{\text {trend }}$ & & $<0.0001$ & & 0.0799 & & $<0.0001$ & & $<0.0001$ \\
\hline$P_{\text {effect }}$ & \multicolumn{4}{|c|}{0.0003} & \multicolumn{4}{|c|}{$<0.0001$} \\
\hline$P_{\text {interaction }}$ & \multicolumn{4}{|c|}{$<0.0001$} & \multicolumn{4}{|c|}{0.0002} \\
\hline
\end{tabular}

Abbreviations: BMI, body mass index; Cl, confidence interval; ITT, intention-to-treat; PP, per-protocol; SBP, systolic blood pressure.

The logistic mixed models were employed to adjust for baseline SBP, age, BMI and the duration of hypertension. $P_{\text {trend: }} P$-values for the linear trend of time of the adjusted hypertension control rate in intervention and control groups, separately. $P_{\text {effect }}: P$-values for the overall effect of intervention in the study period. $P_{\text {interaction: }} P$-values for the difference of slopes of time linear trend

between intervention and control. ${ }^{*}$ The significance between intervention and control groups in each time points: $0.01<P<0.05 ; * \star * P<0.01$.

a Number in the brackets are sample size for ITT analysis/for PP analysis.

became a major reason why awareness, treatment and control of hypertension were low and changed little in China. ${ }^{7}$ To address this problem affordably, particularly in low-income settings, primary healthcare providers must be mobilized to take the frontline role in managing hypertensive patients living in their communities, to better control hypertension at the population level. For this reason, the Primary Healthcare Practice Guidelines for Hypertension Prevention and Control was developed. However, there was some doubt that the Practice Guidelines would be applicable and effective, considering the general lack of professional medical training of primary healthcare providers, particularly in rural China.

The present study demonstrated that the application of customized Practice Guidelines significantly raised the control of blood pressure by more than $50 \%$ in urban primary care and by more than $200 \%$ in rural primary care in Beijing. We also noted significantly larger mean reductions of SBP and DBP in patients in the intervention communities. The results from PP and ITT analyses were very similar, indicating the missing follow-up visits did not affect the study results significantly. Patients in the intervention groups received more advice on therapeutical lifestyle changes and were more likely to receive combination drug therapy, with significantly less cost. These results showed that the Practice Guidelines are applicable and effective in the control of hypertension in China. More importantly, the high control rate attained in intervention groups, both urban and rural, demonstrated that the Chinese primary healthcare providers could be the frontline for management and control of hypertension. Finally, the economic analysis indicates that the intervention is cost-saving with initial investment in training, administration and staff time more than offset by savings in drug expenditure.

Previous studies found a strategy of healthcare provider education alone led to modest or negative results in hypertension control. ${ }^{14-20}$
However, the effect of healthcare provider education varied in the level of intensity, such as mailed education material (low intensity), traditional lecture (moderate intensity) and visits to physicians' offices by peers (high intensity). ${ }^{14,21-24}$ The intensity of our intervention was high, as we provided a quarterly, individual-based consolidated training session following the initial group training, which not only ensured the compliance and the capability of the providers in hypertension management, but also indirectly strengthened the doctor-patient relationship after the patient's health improved. ${ }^{25-32}$ Higher percentage of study patients who complied with follow-up visits in the study in interventions than in controls, $76 \%$ (107/140) vs. $60 \%(84 / 140)$ for urban and $94 \%(132 / 140)$ vs. $83 \%$ (116/140) for rural, indicates that the intervention also increased the use of primary healthcare services by patients. This was possibly mediated through an increase in levels of trust in the primary healthcare providers developed through the intervention.

The effect of implementing clinical guidelines in the management of hypertension in general practice has been evaluated by others. However, the effect reported was much smaller than the present study. For example, Hetlevik et al. ${ }^{33}$ reported that using a computer-based clinical decision support system and a specific implementation strategy resulted in $1 \mathrm{~mm} \mathrm{Hg}$ of $\mathrm{DBP}$ and $1.2 \mathrm{~mm} \mathrm{Hg}$ of SBP in favor of the intervention group after 18 months.

The possible reasons for the very significant increase in control of blood pressure in our study include the following. First, the Practice Guidelines were customized to the providers and the existing availability of medications and laboratory tests in the Chinese primary healthcare facilities. This ensured the acceptability and applicability of the intervention for grass-root level doctors. Second, in contrast to the situation in San Francisco, the baseline knowledge and capability of the Chinese primary healthcare providers in management of 
Table 3 Unadjusted and adjusted mean reduction $(95 \% \mathrm{Cl})$ in SBP and DBP $(\mathrm{mm} \mathrm{Hg})$ in intervention and control groups, urban and rural, at different time of follow-up, adjusting for the baseline blood pressure, age, BMI and duration of hypertension

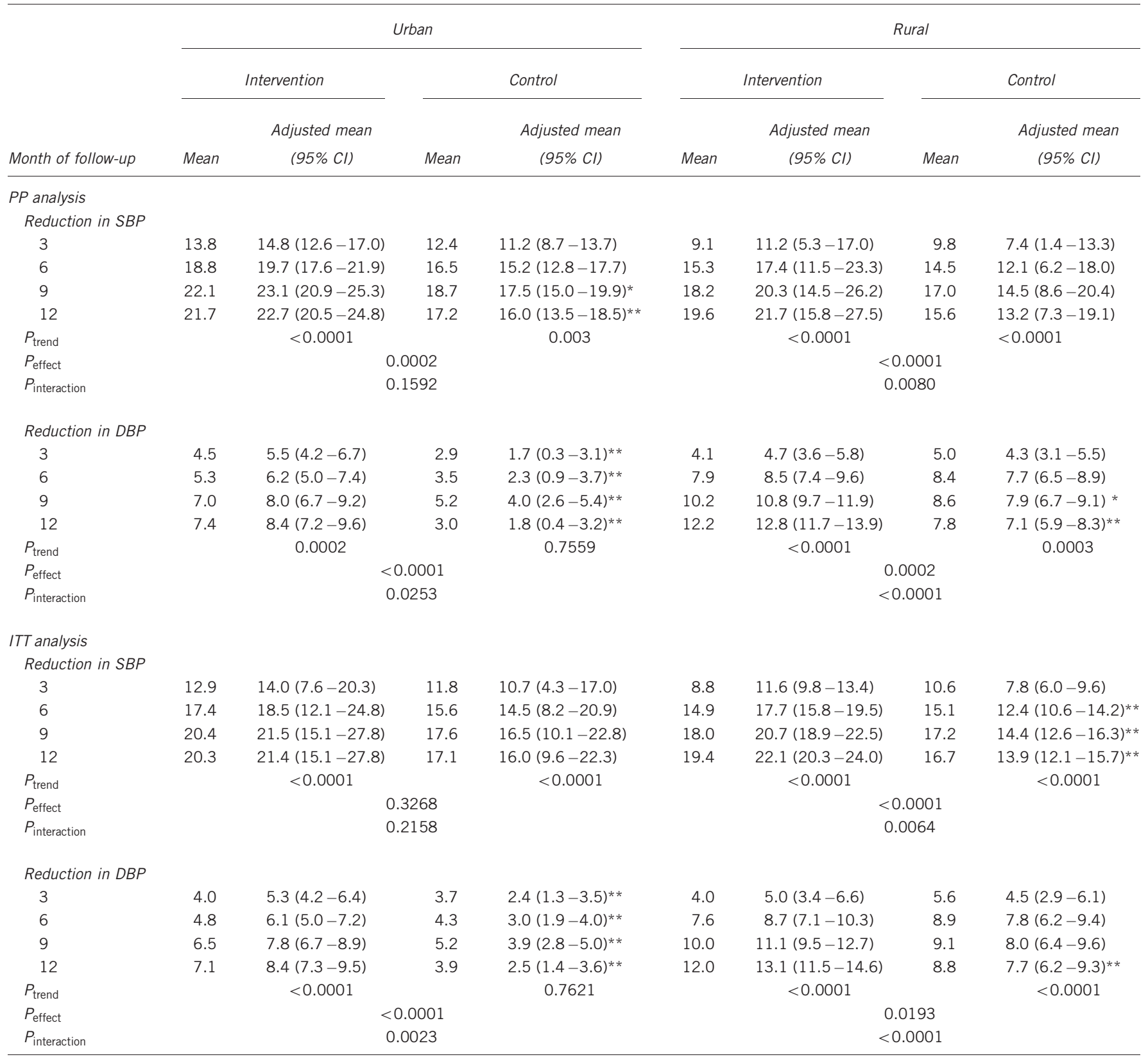

Abbreviations: BMI, body mass index; Cl, confidence interval; DBP, diastolic blood pressure; ITT, intention-to-treat; PP, per-protocol; SBP, systolic blood pressure.

The general mixed models were employed to adjust for baseline blood pressure, age, BMI and the duration of hypertension. $P$ trend: $P$-values for the linear time trend of adjusted mean reduction of SBP/DBP in intervention and control groups, separately. $P_{\text {effect }} P$-values for the overall effect of intervention in the study period. $P_{\text {interaction }}: P$-values for testing the difference on the slope of reduction of blood pressure levels over months of follow-up. ${ }^{*}$ The significance between intervention and control groups in each time points: $0.01<P<0.05$; ${ }^{* *} P<0.01$.

hypertension was much lower, as demonstrated in the control groups, giving larger room for improvement. ${ }^{15,34}$

Results showed that patients in the intervention group generally spent less money on drugs, a potentially counterintuitive result, given that the aim of the intervention is to promote hypertension control. This is because the guidelines recommended the primary healthcare providers to first use the low-cost generic drugs instead of those expensive new drugs as one of the principles for pharmaceutical treatment. Thus, although the number of drugs might have increased, cost-savings were achieved through more cost-effective prescribing. In China, the price for the most expensive anti-hypertension medication per day is over 100 time of the cheapest one, with the cost often borne out-of-pocket by patients. This finding, thus, indicates not only the potential public health benefit through adherence to blood pressure control guidelines but also the scope for significant cost-savings to individuals and the health sector.

Our study has the following limitations. First, we were not able to recruit more community health centers to ensure better representation because of resource constraints. However, we were careful in the selection of the community health centers through consultation with the local health authorities to ensure they are as typical as possible. We also randomized the intervention and control groups, although 
Table 4 The costs and cost-effectiveness in each group, urban and rural

\begin{tabular}{|c|c|c|c|c|}
\hline \multirow[b]{2}{*}{ Analysis } & \multicolumn{2}{|c|}{ Urban } & \multicolumn{2}{|c|}{ Rural } \\
\hline & Intervention & Control & Intervention & Control \\
\hline \multicolumn{5}{|l|}{ ITT analysis } \\
\hline \multicolumn{5}{|l|}{ Cost (\$) } \\
\hline Anti-hypertensive drugs & 8964.1 & 13241.0 & 5082.4 & 7515.1 \\
\hline Salary costs of primary healthcare providers & 379.3 & 223.1 & 227.5 & 133.9 \\
\hline CMR & 50.7 & 50.7 & 50.7 & 50.7 \\
\hline Total (\$) & 11097.6 & 13940.1 & 7257.5 & 8231.3 \\
\hline Average cost per patient $(\$)$ & 79.3 & 99.6 & 51.8 & 58.8 \\
\hline Incremental cost per patient $(\$)$ & & -20.3 & & -7.0 \\
\hline \multicolumn{5}{|l|}{$P P$ analysis } \\
\hline Administration & 797.4 & 425.3 & 894.0 & 531.6 \\
\hline CMR & 38.8 & 30.4 & 47.8 & 41.0 \\
\hline Total (\$) & 9163.8 & 9608.2 & 7080.9 & 7171.3 \\
\hline Average cost per patient $(\$)$ & 85.6 & 114.4 & 53.6 & 63.5 \\
\hline Incremental cost per patient $(\$)$ & & -28.7 & & -9.8 \\
\hline
\end{tabular}

Abbreviations: CMR, case management records; ITT, intention-to-treat; PP, per-protocol.

The exchange rate for RMB (Ren Min Bi) against 100\$ was 827.72 in 2002.

Table 5 Percentage (\%) of patients receiving TLC consultation based on CMR, and pharmacological treatments based on prescription records, provided by primary healthcare providers, when followed up at the first month

\begin{tabular}{|c|c|c|c|c|}
\hline & \multicolumn{2}{|c|}{ Urban } & \multicolumn{2}{|c|}{ Rural } \\
\hline & Intervention & Control & Intervention & Control \\
\hline \multicolumn{5}{|l|}{ TLC consultation } \\
\hline Healthy diet & $68.1^{*}$ & 0.7 & $38.8^{*}$ & 13.4 \\
\hline Salt reduction & $76.9 *$ & 0.7 & $66.7^{*}$ & 9.4 \\
\hline Restricted alcohol intake (drinkers) & $59.3^{*}$ & 0.0 & $61.5^{*}$ & 0.0 \\
\hline Weight reduction (overweight/obese) & $46.4^{*}$ & 0.9 & $30.5^{*}$ & 5.9 \\
\hline Physical activity & $72.5^{*}$ & 0.0 & $56.5^{*}$ & 9.4 \\
\hline Smoking cessation (smokers) & $57.1^{*}$ & 5.6 & $15.6^{*}$ & 0.7 \\
\hline Psychological balance & $25.6^{*}$ & 0.0 & $19.7 *$ & 4.0 \\
\hline \multicolumn{5}{|l|}{ Pharmacological treatments } \\
\hline Calcium antagonists & $67.9 *$ & 48.6 & $30.7 *$ & 55.0 \\
\hline Polypill & $21.4^{*}$ & 49.3 & $72.1^{*}$ & 63.6 \\
\hline ACEI/ARB & $30.7^{*}$ & 14.3 & $9.3^{*}$ & 25.0 \\
\hline Diuretics & $15.7^{*}$ & 4.3 & $8.6^{*}$ & 2.1 \\
\hline$\beta$-blocker & 14.3 & 26.4 & $8.6^{*}$ & 5.0 \\
\hline \multicolumn{5}{|l|}{ Combination } \\
\hline Single-drug therapy & 57.1 & 52.9 & 64.3 & 49.3 \\
\hline 2-drug combination therapy & 29.3 & 41.4 & 32.1 & 42.1 \\
\hline 3-drug combination therapy & 13.6 & 5.7 & 3.6 & 8.6 \\
\hline
\end{tabular}

Abbreviations: ACEI, angiotensin-converting enzyme inhibitors; ARB, angiotensin II receptor antagonists; CMR, case management records; TLC, therapeutic lifestyle change. Note: ${ }^{*} P<0.05$. only two centers were available in each urban and rural setting. However, the comparison of baseline characteristics showed that patients in the interventions had significantly lower SBP and DBP than in the controls, which may lead to a better control of blood pressure. The difference at the end of 12 months between intervention and control, both in control of hypertension and in reduction of blood pressure, existed after adjustment for the baseline blood pressure as well as other potential confounders, employing the multiple regression models. Second, as the study was quasi-experimental study and only one cluster was included in each arm, we could not fully eliminate the possibility of chance or other factors as the cause of the effect. But to minimize this issue, we selected the study sites from both urban and rural areas, and had parallel control for both urban and rural areas in the study. The significant increase in blood pressure control took place in both urban and rural intervention groups, strengthening the link between the results and the intervention. Furthermore, we measured the effects every 3 months and demonstrated that an increase in blood pressure control in the control groups took place only in the first 3 months, but did not continue in the later follow-ups, suggesting that the effect was coming from the intervention rather than just by chance or other factors. Third, we could not blind the measurers of blood pressure (the primary healthcare providers) in an open-label trial such as this. However, we tried to minimize the possible bias by using a uniform protocol in the training all the primary healthcare providers and through the use of electronic devices for the measurement of blood pressure. Fourth, we did not collect baseline data on pharmaceutical and non-pharmaceutical treatments, as the patient recruitment was done after the initial training. The lack of such information at baseline could weaken our inference on the causal relationship 


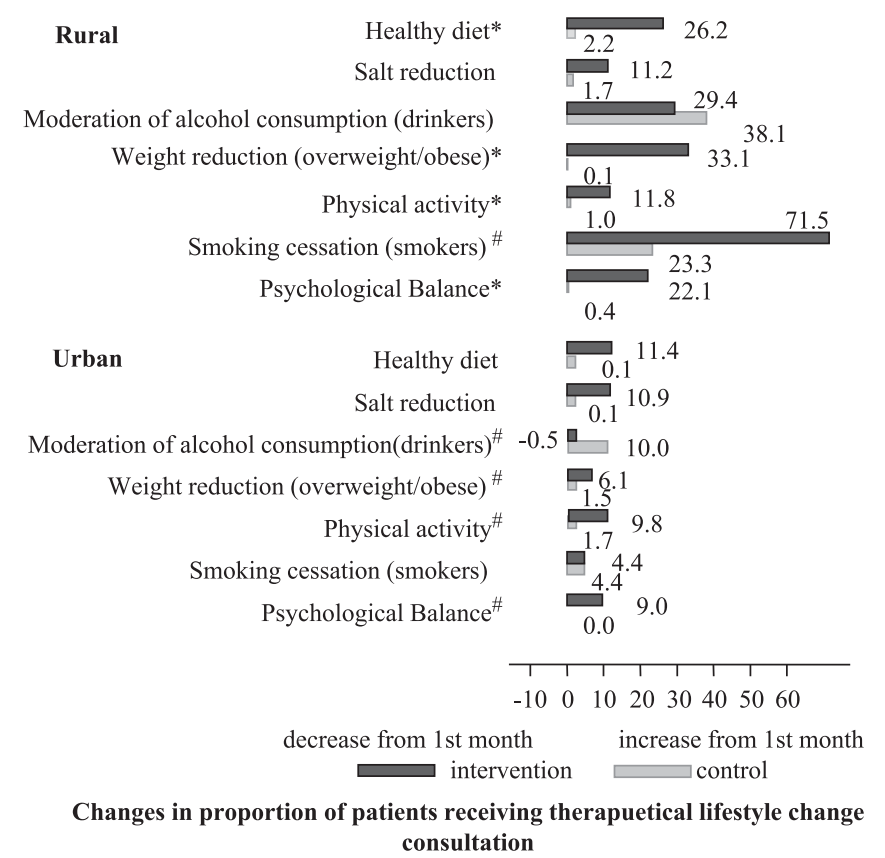

Figure 2 Absolute changes in proportion of patients receiving therapeutical lifestyle change consultation (left) and pharmacological treatments (right) from month 1 to month 12 of study period in intervention and control. The logistic mixed models were employed to compare between intervention and control groups the trend in changes from month 1 to month $12 .{ }^{*} P<0.05$; \# indicates model that does not converge. ACEl, angiotensin-converting enzyme inhibitors; ARB, angiotensin II receptor antagonists. between the intervention and effect. However, our findings that the percentage of patients on non-pharmacological treatments and on combination drug therapy increased significantly in the intervention from month 1 to month 12, but not in controls, strongly support the conclusion that doctor and patient behavior changes were true and led to the effect of intervention. Finally, we collected data for the cost of blood pressure-lowering agents only, but not other treatments for cardiovascular and renal diseases due to the resource constraints. Although the intervention effects and potential costs and cost-savings may have had a secondary influence beyond blood pressure-lowering agents, we had no direct evidence available to estimate these.

In conclusion, our findings provide evidence that application of customized guidelines could effectively improve management of hypertension in both urban and rural community health centers in China, although resulting in cost-savings. This study has substantial implications for hypertension management in primary healthcare in China.

\section{CONFLICT OF INTEREST}

The authors declare no conflict of interest.

\section{ACKNOWLEDGEMENTS}

We are grateful to all the study patients for their contribution to the study. We thank all primary healthcare providers working at the four study centers: Yinghai Community Health Center and Taihe Community Health Center, Daxing, Beijing; Fengbo Community Health Center, Shunyi, Beijing; Ganjiakou Community Health Center, Xicheng, Beijing; and Jinsong Community Health Center, Jinsong, Beijing, for their active participation and implementation of the study, particularly Dr Jianhua Qi, Baojun Chen, Bing Li, Zhigang Yang, Huifu Bai, Zhimin Ma and Naili Zheng for their great leadership in implementation of the study in the centers. We thank Ms Danielle Millican at The George Institute for Global Health, Australia, for her assistance in editing the manuscript.

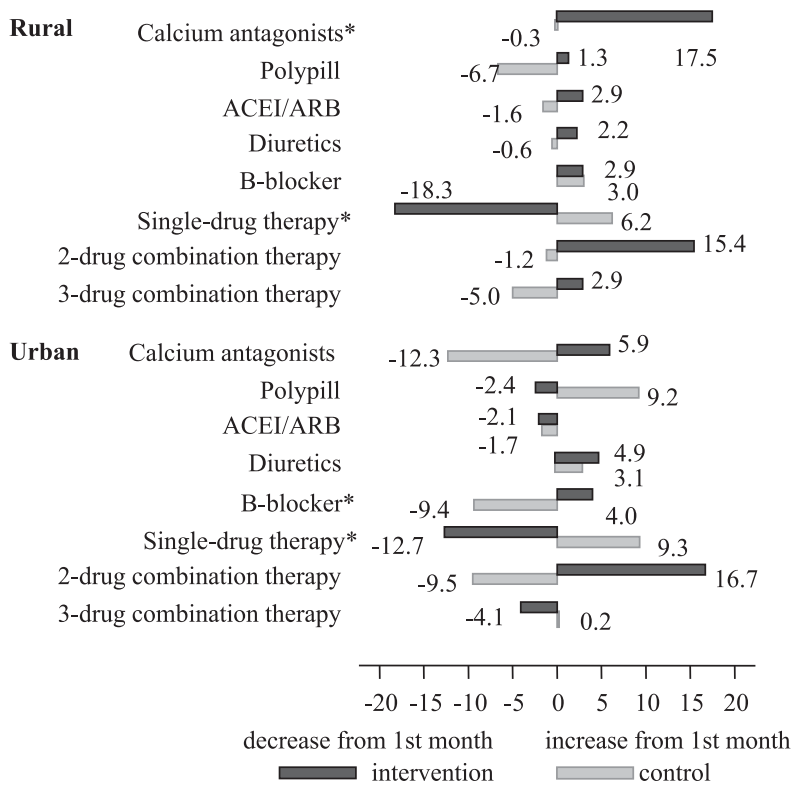

Changes in proportion of patients receiving pharmacological treatments
1 Wang L. Comprehensive Report, Chinese Nutrition and Health Survey in 2002 (People's Medical Publishing House; Beijing, China, 2005).

2 Wang L, Kong L, Wu F, Bai Y, Burton R. Preventing chronic diseases in China. Lancet 2005; 366: 1821-1824.

3 Yang G, Kong L, Zhao W, Wan X, Zhai Y, Chen LC, Koplan JP. Emergence of chronic non-communicable diseases in China. Lancet 2008; 372: 1697-1705.

4 He J, Gu D, Wu X, Reynolds K, Duan X, Yao C, Wang J, Chen CS, Chen J, Wildman RP, Klag MJ, Whelton PK. Major causes of death among men and women in China. N Engl J Med 2005; 353: 1124-1134.

5 Lopez AD, Mathers CD, Ezzati M, Jamison DT, Murray CJ. Global and regional burden of disease and risk factors, 2001: systematic analysis of population health data. Lancet 2006; 367: 1747-1757.

6 Liu L, Gong L. China Guidelines on Prevention and Management of High Pressure (2005 Revision), 2005.

7 Wu Y, Huxley R, Li L, Anna V, Xie G, Yao C, Woodward M, Li X, Chalmers J, Gao R, Kong $L$, Yang X. Prevalence, awareness, treatment, and control of hypertension in China: Data from the China National Nutrition and Health Survey 2002. Circulation 2008; 118: 2679-2686.

8 Liu L, Wang W, Yao C. China Guidelines on Prevention and Management of High Pressure (2009 Primary-care). (People's Medical Publishing House; Beijing, China, 2009).

9 Chobanian AV, Bakris GL, Black HR, Cushman WC, Green LA, Izzo Jr JL, Jones DW, Materson BJ, Oparil S, Wright Jr JT, Roccella EJ. The seventh report of the Joint National Committee on Prevention, Detection, Evaluation, and Treatment of High Blood Pressure: the JNC 7 Report. Hypertension 2004; 42: 1206-1252.

10 Yusuf S, Reddy S, Ounpuu S, Anand S. Global burden of cardiovascular diseases, part I: general considerations, the epidemiologic transition, risk factors, and impact of urbanization. Circulation 2001; 104: 2746-2753.

11 Wu Y, Yan X, Yao C, Fang X. Practice Guidelines on Prevention and Treatment of Hypertension for Primary Care Physicians. Chinese J Gen Pract 2003; 2: 1-4.

$12 \mathrm{Li} \mathrm{L,} \mathrm{Rao} \mathrm{K,} \mathrm{Kong} \mathrm{L,} \mathrm{Yao} \mathrm{C.} \mathrm{A} \mathrm{description} \mathrm{on} \mathrm{the} \mathrm{Chinese} \mathrm{national} \mathrm{nutrition} \mathrm{and} \mathrm{health}$ survey in 2002. Chinese J Epidemiol 2005; 7: 478-484.

13 Zhou BF, Wu XG. Manual of Method for Epidemiology Survey of Cardiovascular Disease. Beijing Medical University and Peking Union Medical College United Press; Beijing, China, 1997.

14 Dickinson JC, Warshaw GA, Gehlbach SH, Bobula JA, Muhlbaier LH, Parkerson Jr GR. Improving hypertension control: impact of computer feedback and physician education. Med Care 1981; 19: 843-854.

15 Gullion DS, Tschann JM, Adamson TE, Coates TJ. Management of hypertension in private practice: A randomized controlled trial in continuing medical education. J Contin Educ Health Prof 1988; 8: 239-255.

16 Davis D. Physician education, evidence and the coming of age of CME. J Gen Intern Med 1996; 11: 705-706.

17 Davis D. Does CME work? An analysis of the effect of educational activities on physician performance or health care outcomes. Int J Psychiatry Med 1998; 28: 21-39. 
18 Avanzini F, Corsetti A, Maglione T, Alli C, Colombo F, Torri V, Floriani I, Tognoni G. Simple, shared guidelines raise the quality of antihypertensive treatment in routine care. Am Heart J 2002; 144: 726-732.

19 Grimshaw JM, Eccles MP, Walker AE, Thomas RE. Changing physicians' behavior: what works and thoughts on getting more things to work. J Contin Educ Health Prof 2002; 22: 237-243.

20 Walsh JM, McDonald KM, Shojania KG, Sundaram V, Nayak S, Lewis R, Owens DK, Goldstein MK. Quality improvement strategies for hypertension management: a systematic review. Med Care 2006; 44: 646-657.

21 Linn BS. Continuing medical education: impact on emergency room bum care. JAMA 1980; 244: 565-570.

22 Evans CE, Haynes RB, Birkett NJ, Gilbert JR, Taylor DW, Sackett DL, Johnston ME, Hewson SA. Does a mailed continuing education program improve physician performance? Results of a randomized trial in antihypertensive care. JAMA 1986; 255: 501-504.

23 Ray WA, Blazer DG, Schaffner W, Federspiel CF, Fink R. Reducing long-term diazepam prescribing in office practice: a controlled trial of educational visits. JAMA 1986; 256 : 2536-2539.

24 Jafar TH, Hatcher J, Poulter N, Islam M, Hashmi S. Community-based interventions to promote blood pressure control in a developing country. Ann Intern Med 2009; 151: 593-601.

25 Davis D. Clinical practice guidelines and the translation of knowledge: the science of continuing medical education. CMAJ 2000; 163: 1278-1279.
26 Wagner EH, Austin BT, Davis C, Hindmarsh M, Schaefer J, Bonomi A. Improving chronic illness care: translating evidence into action. Health Aff (Millwood) 2001; 20: 64-78.

27 Wagner TH, Hu TW, Hibbard JH. The demand for consumer health information. $J$ Health Econ 2001; 20: 1059-1075.

28 Bodenheimer T, Wagner EH, Grumbach K. Improving primary care for patients with chronic illness: the chronic care model, Part 2. JAMA 2002; 288: 1909-1914.

$29 \mathrm{Hibbard} \mathrm{JH}$. Engaging health care consumers to improve the quality of care. Med Care 2003; 41: 161-170.

30 Godfrey MM, Nelson EC, Wasson JH, Mohr JJ, Batalden PB. Microsystems in health care: part 3. Planning patient-centered services. Jt Comm J Qual Patient Saf 2003; 29: $159-170$.

31 Kassirer JP. The quality of care and the quality of measuring it. N Engl J Med 1993; 329: 1263-1265.

32 Bodenheimer T. Interventions to improve chronic illness care: evaluating their effectiveness. Dis Manag 2003; 6: 63-71.

33 Hetlevik I, Holmen J, Kruger O. Implementing clinical guidelines in the treatment of hypertension in general practice. Evaluation of patient outcome related to implementation of a computer-based clinical decision support system. Scand J Prim Health Care 1999; 17: 35-40.

34 Wu Y, Wang Z, Gao R. A survey on perception and knowledge of Chinese health care professionals in prevention and control of hypertension. Chinese J Cardiovasc Dis 2004; 32: 264-269. 